\title{
'B 663' Possible Anti-inflammatory Action in Lepromatous Leprosy
}

\author{
S. G. BROWNE, M.D., F.R.G.P., F.R.G.S., D.T.M. \\ Leprosy Service Research Unit, Uzuakoli, Eastern Nigeria
}

In the course of the clinical trial of ' $\mathrm{B} 663$ ' (Geigy, S. A.) see Barry, V. C., Conalty, M. L. I 965 Leprosy Review, this issue reported by Browne and Hogerzeil ( I $962 a, b)$, it was noted that only two out of the 26 participating patients suffering from lepromatous leprosy developed any symptoms of acute exacerbation while receiving the drug, and in both instances the attack was slight and transient and occurred during the first month of treatment. It is a well-attested observation that in any group of patients suffering from lepromatous leprosy of comparable severityand receiving dapsone in standard doses for a similar period, a much higher proportion would have developed some degree of acute exacerbation. Of the 26 patients, 2 I had B 663 for six months, and the remaining five for $\mathrm{I} 2$ months: 13 patients received $\mathrm{B} 663$ alone, nine together with dapsone and four with dapsone and ditophal.

(Two patients suffering from bacteriologically positive borderline leprosy are excluded from consideration here, since they are not subject to the same type of acute exacerbation as are patients with lepromatous leprosy. As a matter of interest, however, it may be recorded that neither had any kind of exacerbation during treatment with B 663, but that one of them subsequently developed an acute ulnar neuritis.)

\section{Acute exacerbation on subsequent dapsone therapy}

That this group of 26 patients with lepromatous leprosy is typical in respect of liability to exacerbation is shown by the fact that after dapsone had been substituted for B 663, I 4 of them developed erythema nodosum leprosum (ENL) and various other manifestations of acute exacerbation. In addition, some patients showed certain features that are frequently though not necessarily associated with such a state; thus, three had acute polyneuritis, one had severe bilateral dactylitis, and one passed through an acute psychotic episode. It should be added that one patient succumbed to pneumonia during the i $3^{\text {th }}$ month of treatment for leprosy.

In the I 4 patients affected, the sign of ENL appeared first at various intervals after the change-over to dapsone therpay. In no patient did ENL lesions appear until seven months had elapsed after B 663 therapy had been discontinued. Thus, they appeared after eight to I 2 months in five patients; after I 3 to 18 months in three; after i 9 to 24 months in five, and during the 29th month in one.

To summarize: during treatment with B 663, two patients developed ENL during I 86 patient/ months (i.e. five patients for 12 months, and $2 \mathrm{I}$ patients for six months); no patient developed ENL after the first month of such treatment, i.e. in I6o patient/months. During treatment with dapsone, I 3 patients developed ENL during the next 589 patient/months (i.e. 24 patients for 24 months, plus one patient deceased after i3 months), and one patient developed ENL after 29 months.

The two patients who had experienced a transient attack of ENL during the first month of B 663 treatment did not develop any ENL lesions when subsequently taking dapsone.

As in other reported series, there was no constant correlation between the appearance of ENL and the absolute height of the Bacterial Index, or its increase or decrease, or changes in the proportion of normal-staining bacilli (the Morphological Index).

The occurrence of bacterial 'resistance' in five patients after i 2 months of B 663 therapy alone, as shown by a more or less sudden increase in the Bacterial Index and the simultaneous reappearance of normal solid-staining rods in the smears at several sites (Browne and Hogerzeil i 962 c.), seemed to have no bearing on the incidence of acute exacerbation.

$$
\text { 'B 663' - Anti-inflammatory Action }
$$


Influence of total dose of $B 663$

There was no evidence that the five patients who had received $\mathrm{B} 663$ for $\mathrm{I} 2$ months were less liable to experience acute exacerbation than the $2 \mathrm{I}$ who had received the drug for six months, or that in them the onset of acute exacerbation was postponed by the additional a mount of drug they had received. Three of these five experienced ENL of the same severity as the patients who had received the drug for only six months, and the time of onset appeared to be similarly fortuitous.

\section{Clinical features of acute exacerbation}

There was nothing in the clinical features of the acute exacerbation in these patients to distinguish them from the general run of such cases in West Africa (Browne I963). All grades of severity were noted, from the slight and transient, accompanied by no systemic disturbance, to the severe and persistent, characterized by high temperature, general malaise, polyarthralgia, polyadenitis and widespread neuritis in addition to the cutaneous signs of superficial erythema nodosum and deep diffuse panniculitis. Iritis and iridocyclitis were not observed. Three patients had a single short attack, which cleared up either spontaneously within a month, or during temporary withdrawal of dapsone. In three patients, the first attack was more severe, and persisted for from five to 14 months. Ten patients had recurrent attacks spread over $3^{\circ}$ months. In one patient in whom the acute exacerbation persisted for I 4 months, the skin of the lower part of the posterior aspect of the arms became hard, dark, shiny and tender part of the picture of progressive lepra reaction.

In spite of the occurrence of ENL in these patients, and the consequent short or prolonged interruption of treatment in many instances, their overall clinical and bacteriological progress has been eminently satisfactory.

\section{I G U S S I O N}

The incidence and pattern of ENL in this group of 26 patients (reduced to 25 by death) with lepromatous leprosy under treatment with standard doses of dapsone are in keeping with experiences of similar groups in West Africa. The interest lies in the virtual absence of acute exacerbation during previous treatment with B 663, whether or not another drug was being given concurrently. From subsequent experience, it is apparent that the members of the group were normally prone to exacerbation: they did not experience it while receiving B 663, but did so afterwards.

Since the pathogenesis of acute exacerbation in lepromatous leprosy is still obscure, it is idle at this juncture to speculate on the precise mechanism of B 663 in suppressing the phenomenon.

It is worth recording, however, that the disappearance of morphologically normal bacilli from the skin smears was proceeding at a very satisfactory rate during administration of $\mathrm{B} 663$, and that disintegrating bacilli were present in an ever-increasing proportion of the bacillary load in the dermis and nasal mucosa.

It was not possible, because supplies of the drug were exhausted, to observe the effect of readministration of $\mathrm{B} 663$ on patients who had developed acute exacerbation. Any sustained benefit that might be noted on the established condition would support the suggestion here advanced that B 663 appears somehow to exert a suppressive action on the development of acute exacerbation in lepromatous leprosy.

If it could be shown that patients with lepromatous leprosy under prolonged treatment with B 663, with perhaps the addition of dapsone, make rapid clinical and bacteriological progress and are spared the distress and risks of more or less prolonged episodes of acute exacerbation, with accompanying polyneuritis and ocular complications, then $\mathrm{B} 663$ may well prove to be a drug with a definite and extensive sphere of usefulness in leprosy.

\section{S UM M A R Y}

Erythema nodosum leprosum occurred in only two out of 26 patients whilst they were undergoing treatment for lepromatous leprosy with B 663 (Geigy), and in both cases the condition occurred within the first four weeks of treatment and was slight and transient. When all the patients were subsequently given standard doses of dapsone, however, I 4 of them passed through episodes of erythema nodosum leprosum whose clinical features corresponded with those observed in similar groups. It is suggested that B 663 (Geigy) may exert a suppressive effect on the development of acute exacerbation in lepromatous leprosy.
IO Leprosy Revieus 
A CKNOWLEDGEMENTS

Thanks are expressed to Messrs J. R. Geigy, S.A., for supplies of B 663, and to Dr S. O. Egwuatu, Chief Medical Officer, Ministry of Health, Eastern Nigeria, for permission to publish this article.
References

browne, s. G. (1963). J. chr. Dis., 16, 23.

BRowne, s. G. and hogerzeil, L. m. (i 962a). Leprosy Rev., 33, 6 .

Browne, s. G. and hogerzeil, L. m. (I 962b). Leprosy Rev., 33, I8I.

BROWNe, s. G. and HOGERzeIL, L. M. (I 962c). Leprosy Rev., 33, 185 . 